\title{
Review \\ Genetic Heterogeneity and Taxonomic Diversity among Gardnerella Species
}

\author{
Joana Castro, ${ }^{1}$ Kimberly K. Jefferson, ${ }^{2}$ and Nuno Cerca ${ }^{1, *}$
}

Gardnerella vaginalis has been considered a pivotal player in the progression of bacterial vaginosis (BV), a condition associated with serious health complications. However, G. vaginalis is also commonly found in asymptomatic or BV-negative women. This has generated interest in the question of whether genetic differences among isolates might distinguish pathogenic from commensal isolates. G. vaginalis was the only recognized species in its genus for four decades, but recently an emended description of $G$. vaginalis and descriptions of three new species - Gardnerella leopoldii, Gardnerella piotii, and Gardnerella swidsinskii - have been proposed. This review provides background on the heterogeneity and diversity within the genus Gardnerella, highlighting the main features that distinguish species and clades, and how these features may impact BV development.

\section{Clinical Relevance of Gardnerella in BV}

Worldwide, BV is the most common gynecological disorder among women of childbearing age, affecting approximately $29 \%$ of women in the general population and $50 \%$ of African American women [1-5]. Microbiologically, BV is characterized by a dramatic shift in the vaginal microbiota from the dominant lactic acid and $\mathrm{H}_{2} \mathrm{O}_{2}$-producing bacteria (mainly lactobacilli) to a more taxonomically rich microbiota, consisting of strict and facultative anaerobes [6-8].

Gardnerella spp. have been studied more than any other species in the vaginal ecosystem because it is the dominant microorganism in the vaginal microbiome of many women with $\mathrm{BV}[6,9]$. BV is asymptomatic in half of the cases, but can be associated with malodorous vaginal discharge, increased vaginal $\mathrm{pH}$, and the presence of clue cells $[10,11]$. Swidsinski and colleagues [12], using fluorescence in situ hybridization (FISH) (see Glossary) specific for Gardnerella spp., were the first to show that these species are able to form biofilms on vaginal epithelium in women with BV, explaining the nature of clue cells, that is, cells covered with a biofilm predominantly formed by Gardnerella spp., and as such providing convincing evidence for the etiological role of Gardnerella spp. in this condition. Importantly, even when BV is asymptomatic, it is associated with increased risks for preterm delivery [13], postpartum endometritis [14], pelvic inflammatory disease [15], and inf0ection with human immunodeficiency virus [16].

Over the last four decades, the role of Gardnerella spp. as a pathogen has been disputed because women in whom BV has been clinically ruled out are often colonized with Gardnerella spp. $[17,18]$. Here, we discuss the possibility that confusion about the role of Gardnerella spp. in BV pathogenesis has been exacerbated by prior insufficient taxonomic fine-tuning, and that different species with distinct ecological and/or pathological properties have all been lumped together within the species $G$. vaginalis. As such, in this review, we refer to G. vaginalis only in historical context, and all references to more recent papers are described with the term Gardnerella spp.

\section{Focusing on Heterogeneity and Diversity within Gardnerella}

The original discovery of the genus Gardnerella dates back to 1953, when Leopold described this microorganism as a novel 'Haemophilus-like' species associated with prostatitis and cervicitis [19]. Two years later, Gardner and Dukes described this microorganism in relation to nonspecific vaginitis (a classical name for BV), renaming this bacterium Haemophilus vaginalis [20]. Afterwards, this bacterium was reclassified within the genus Corynebacterium [21]. Later, two large taxonomic studies demonstrated the lack of similarity between this bacterium and other established genera, which resulted in the emergence of the new genus Gardnerella, with its closest relatives found in the genus

\section{Highlights}

Gardnerella spp. are the most frequent organisms found in bacterial vaginosis (BV). However, colonization by Gardnerella spp. does not always lead to BV

Developments in molecular genetics have shed new light on the diversity of the genus Gardnerella, leading to an emended description of Gardnerella vaginalis and, for the first time, the inclusion of more species of Gardnerella, namely, Gardnerella leopoldii, Gardnerella piotii, and Gardnerella swidsinskii.

While no association had been made between the novel Gardnerella species and virulence potential, there is genomic evidence of a higher prevalence of some genotypes in BV cases, as compared with colonization in women in whom BV has been clinically ruled out.

${ }^{1}$ Centre of Biological Engineering (CEB), Laboratory of Research in Biofilms Rosário Oliveira (LIBRO), University of Minho, Campus de Gualtar, 4710-057 Braga, Portugal

2Department of Microbiology and Immunology, Virginia Commonwealth University, Richmond, VA 23298-0678c, USA

*Correspondence:

nunocerca@ceb.uminho.pt 
Bifidobacterium $[22,23]$. At that time, G. vaginalis was the only recognized species in this genus and was described as nonencapsulated, non-spore-forming, pleomorphic rods with an average size of 0.5 to $1.5 \mu \mathrm{m}$ [24]. Despite the fact that it often stains red by the Gram stain, the envelope architecture was definitively shown to be Gram-positive as it lacks an outer membrane [25]. G. vaginalis often produces fimbriae, the role of which may be to mediate its attachment to vaginal epithelial cells [26]. G. vaginalis was commonly known as a facultative anaerobic microorganism and was also described as fastidious, as it grew better at 37으 in complex media in an atmosphere with 5-10\% of carbon dioxide $\left(\mathrm{CO}_{2}\right)$ or in a candle flame extinction jar $[24,27]$. Nevertheless, it was demonstrated that certain $G$. vaginalis strains were strict anaerobes [28]. Finally, biochemical tests revealed that $G$. vaginalis was catalase-, oxidase-, and $\beta$-glucosidase-negative [24].

The terms heterogeneity and diversity are often used interchangeably; however, heterogeneity refers to variability within an integrated entity, whereas diversity implies an entity in which the components diverge or members of different groups occur together [29]. Therefore, if the genus Gardnerella is composed of distinct species, then the genus would be more accurately described as both heterogeneous and diverse. Recently, developments in molecular genetics have shed new light on the genetic heterogeneity and taxonomic diversity within the genus Gardnerella. Notably, experiments on wholegenome sequence analysis were carried out in 81 Gardnerella strains by Vaneechoutte and colleagues in 2019 [30]. These researchers pointed out the existence of at least 13 groups distinct enough to be classified as separate species, within the taxon formerly known as G. vaginalis [30]. It was an important breakthrough in the field that resulted in an emended description of $G$. vaginalis and, for the first time, the inclusion of more Gardnerella species, namely G. leopoldii, G. piotii, and G. swidsinskii [30]. Table 1 shows the common features among the species as well as the main differences, as described by the authors. Interestingly, in blood plates, all colonies were found to be indistinguishable, but the authors described differences relative to $\beta$-galactosidase activity, sialidase activity, and DNA G+C content. Importantly, similar to what is observed for other species [31], Gardnerella spp. cannot be delineated based on the 16S rRNA gene since the 81 genomes analyzed by Vaneechoutte et al. indicated that none of these sequences showed less than 98.5\% similarity [30]. Of note, while $97 \%$ similarity within the $16 \mathrm{~S}$ rRNA sequence is often considered the cut-off for distinguishing bacterial species, the $16 \mathrm{~S}$ rRNA gene sequence is not a component of the formal definition of a species [32].

\section{The Dilemma of Vaginal Colonization by Gardnerella spp. in Asymptomatic or BV-Negative Women}

The strong correlation between BV and Gardnerella spp. has sometimes been taken as direct evidence of causation of BV [33]. Nevertheless, Gardnerella spp. vaginal colonization does not always lead to BV [34]. In fact, Gardnerella spp. often form a constituent of the vaginal microbiota of healthy, asymptomatic women of all ages $[35,36]$. Therefore, the question of how, as an opportunistic pathogen, Gardnerella spp. can exist in healthy asymptomatic women needs resolution so that the role of Gardnerella spp. in BV pathogenesis can be properly understood. One possibility is that only certain lineages or species of Gardnerella are pathogenic and others are natural commensals. Another nonexclusive hypothesis is that some Gardnerella spp. are virulent only when specific conditions arise [37]. To examine these hypotheses, many efforts have been made to decipher the features associated with virulence, using a variety of techniques that characterize the diversity within Gardnerella spp., analyzing the phenotypic properties, genotyping differences, pangenome, ecotypes, and in vitro functional properties.

\section{Biotyping of Gardnerella spp.}

For more than 30 years, the scientific community has been conducting a wide variety of bacterial typing assays, in order to find the possible factors which might lead to different virulence traits among isolates of Gardnerella spp., as described in Table 2.

The phenotypic diversity of the isolates of Gardnerella spp. is well established and has been used as the basis for classification systems whereby isolates were divided into biotypes based on their
Glossary

Amplified ribosomal DNA restriction analysis (ARDRA): a technique based on the DNA sequence variations present in the PCR-amplified 16S rRNA genes. In ARDRA analysis, the PCR product amplified from environmental DNA is generally digested with tetracutter restriction endonucleases, and restricted fragments are resolved on agarose or polyacrylamide gels. Patterns obtained from several restriction enzymes can be compared with those obtained from reference strains to assign a particular isolate to a genotype group. Biofilms: communities of adhering bacteria forming robust layers on abiotic and biotic surfaces. The adherent cells are surrounded by a self-produced matrix of extracellular polymeric substances. Biofilm formation is a major clinical issue because microorganisms within biofilms are difficult to eradicate.

Ecotype: a subgroup within a species that has adapted genotypically and/or phenotypically to the conditions unique to its habitat.

Fluorescence in situ hybridization (FISH): a technique that uses fluorescent-labeled probes to target species-specific rRNA sequences in a highly sensitive and specific fluorescence in situ hybridization assay.

Lysogenic phage: a bacteriophage that is integrated in the bacterial genome, without causing cell lysis.

Lytic phage: a bacteriophage that has the ability to cause bacterial cell lysis.

Pangenome: the entire gene set of all strains of a species; it includes genes present in all strains (core genome) and genes present in only some strains of a species (variable or accessory genome). 
Table 1. Main Features of Known Gardnerella spp. According to Vaneechoutte et al. [30],a

\begin{tabular}{|l|l|l|l|l|}
\hline Feature & $\begin{array}{l}\text { Gardnerella } \\
\text { vaginalis }\end{array}$ & $\begin{array}{l}\text { Gardnerella } \\
\text { leopoldii }\end{array}$ & $\begin{array}{l}\text { Gardnerella } \\
\text { piotii }\end{array}$ & $\begin{array}{l}\text { Gardnerella } \\
\text { swidsinskii }\end{array}$ \\
\hline $\begin{array}{l}\text { B-galactosidase } \\
\text { activity }\end{array}$ & Positive & Negative & Negative & Negative \\
\hline Sialidase activity & Positive/negative & Negative & Positive & Negative \\
\hline DNA G+C content & $\begin{array}{l}41.0 \text { mol\% to } \\
42.8 \text { mol\% }\end{array}$ & $\begin{array}{l}41.9 \text { mol\% } \\
\text { to } 43.2 \text { mol\% }\end{array}$ & $\begin{array}{l}41.1 \text { mol\% to } \\
42.3 \text { mol\% }\end{array}$ & $\begin{array}{l}41.4 \text { mol\% to } \\
42.3 \text { mol\% }\end{array}$ \\
\hline Colonies & Pinpoint white- & Pinpoint white- & Pinpoint white- & Pinpoint white- \\
\hline
\end{tabular}

${ }^{a}$ The reclassification of the Gardnerella species was performed by matrix-assisted laser desorption/ionization (MALDI)TOF (time of flight) protein profiling [30].

biochemical properties, namely, production of $\beta$-galactosidase, lipase, and hippurate hydrolysis [3841]. Attempts have been made to correlate these biotypes with BV, with one study finding that lipasepositive isolates were more frequently isolated from women with BV than from those without BV [41]. However, other studies found no association between any specific biotype and BV [38,39].

\section{Genotyping of Gardnerella spp.}

Attempts at genotypic differentiation of Gardnerella spp. by amplified ribosomal DNA restriction analysis (ARDRA) into biotypes with specific virulence factors have also yielded variable results and fail to consistently implicate a particular biotype with BV $[42,43]$. Overall, there has been little success in reconciling the genotypic and phenotypic characteristics with each other, or in identifying patterns of association of any genotype or phenotype with demographic or clinical characteristics [42-44].

More recently, the advent of culture-independent methods for determining the composition of the vaginal microbiome, based on whole-genome sequencing, has provided an unprecedented opportunity to investigate the diversity of Gardnerella spp. [45]. Efforts to exploit whole-genome sequencing of isolates of Gardnerella spp. showed disparities in virulence potential among the isolates [46-48]. Although the results of these comparative genomics studies revealed some evidence regarding the distribution of genes responsible for virulence-associated traits, such as cytotoxicity [47] and degradation of mucus [46], conclusions were limited by the small number of isolates studied. As far as we know, vaginolysin and sialidase have been the most extensively studied virulence factors in Gardnerella spp. [49-51]. Vaginolysin is a pore-forming toxin that is able to induce lysis of human erythrocytes, epithelial cells, and polymorphonuclear leukocytes [52,53], whereas sialidase is associated with the degradation of diverse key mucosal protective factors, such as mucins, and also contributes to the exfoliation and detachment of vaginal epithelial cells [54,55]. Importantly, very recently, new insights into the enzymes responsible for sialidase activity have shown that Gardnerella spp. can possess three sialidases: sialidase A (also named neuraminidase or $\mathrm{NanH} 1$ ), $\mathrm{NanH} 2$, and $\mathrm{NanH} 3$ [56]. Of note is that $\mathrm{NanH} 2$ and $\mathrm{NanH} 3$ seem to be the primary sources of sialidase activity in Gardnerella spp. [56].

In an early study of the vaginal microbiome, based on PCR amplification and sequencing of the 'universal target' region of the gene encoding the $60 \mathrm{kDa}$ chaperonin (cpn60), Hill and colleagues [57] described four different subgroups of Gardnerella spp. The same four subgroups were observed in a much larger study of vaginal microbiomes of African women [58]. Jayaprakash et al. [42] subsequently reported that whole-genome average nucleotide identity values between cpn60-defined subgroups were less than 95\% [42]. Confirmation that cpn60-based subdivisions of Gardnerella spp. were not the result of a PCR artifact was supported by a whole-genome sequencing study of 17 strains of Gardnerella [48]; this study revealed that Gardnerella is highly heterogeneous and taxonomically diverse, with only $52 \%$ of the genome of each isolate consisting of conserved genes [48]. 
Table 2. Studies of Gardnerella spp. Differentiation Using Biotyping Approaches

\begin{tabular}{|l|l|l|l|}
\hline Technique & $\begin{array}{l}\text { Number }(n) \text { of } \\
\text { isolates }\end{array}$ & Main conclusion & Refs \\
\hline Biotyping & $n=359$ & $\begin{array}{l}\text { 8 biotypes were found } \\
\text { No significant differences in } \\
\text { biotype distribution }\end{array}$ \\
\hline $\begin{array}{l}\text { Detection of hippurate } \\
\text { hydrolysis, } \beta \text {-galactosidase } \\
\text { and lipase; fermentation of } \\
\text { arabinose, galactose and xylose }\end{array}$ & $n=197$ & $\begin{array}{l}\text { 17 biotypes were found } \\
\text { No significant differences in } \\
\text { biotype distribution }\end{array}$ & [39] \\
\hline $\begin{array}{l}\text { betiotypes were found } \\
\text { Significant differences in } \\
\text { biotype distribution, suggesting } \\
\text { that some biotypes were } \\
\text { associated with BV }\end{array}$ & [40] \\
\hline $\begin{array}{l}\text { Detection of hippurate hydrolysis, } \\
\text { activity with oleate as a substrate }\end{array}$ & $n=261$ & $\begin{array}{l}\text { Significant differences in biotype } \\
\text { distribution, with the lipase-positive } \\
\text { biotypes (biotypes 1, 2, 3, and 4) being } \\
\text { more predominant in women with BV }\end{array}$ & [41] \\
\hline
\end{tabular}

Based on these findings, isolates of Gardnerella spp. were clustered into four subgroups/clades with distinct genomic properties. Notably, the reconciliation of the cpn60 typing [42] and the four subgroups/clades [48] was further proposed in a recent study carried out by Schellenberg and colleagues [59], where cpn60 subgroups A, B, C, and D were shown to correspond to clades 4, 2, 1, and 3, respectively. However, Janulaitiene and colleagues showed that some isolates of Gardnerella spp. did not belong to any clade detectable by clade-specific PCR [60]. Importantly, the establishment of phenotypic properties that differentiate the four subgroups is so far limited to the observation that all subgroup B isolates (and only some subgroup C isolates) are sialidase-activity positive [54,59]. Curiously, the subgroup $C$ isolates might also exhibit lipase activity [42]. Very recently, Vaneechoutte and colleagues [30] showed that clade 1 described by Ahmed et al. [48] contains two species, of which they described one as $G$. vaginalis but the second species was not further characterized. According to the same study, clade 2 also contains two species, one being described as G. piotii, while the other was also undefined. Clade 3 contains three undefined species, while clade 4 contains two species, which they described as G. leopoldii and G. swidsinskii. Figure 1 shows the association between data provided by these whole-sequencing genome studies. Of note, the different conclusions obtained from the studies developed by Ahmed et al. [48] and Vaneechoutte et al. [30] might be explained by the fact that Ahmed and colleagues' analysis was based upon visual inspection of a phylogenetic tree constructed from core gene allelic data, whereas Vaneechoutte and coworkers based their conclusions on full genome similarity statistics.

Importantly, the development of cpn60-based molecular profiling, as well as the clade-specific PCR assays, allowed investigation of the prevalence of each subgroup in vaginal specimens $[17,61-64]$. In this regard, a study employing multitarget quantitative PCR with subgroup-specific primers detected multiple subgroups in $70 \%$ of 60 vaginal samples examined [17]. This is particularly relevant given that multiclade communities of Gardnerella spp. showed a positive association with BV, suggesting that women with BV were colonized with multiple strains of Gardnerella spp. [60,63,65].

\section{Prophage Increases the Breadth of the Gardnerella spp. Pangenome}

The development of advanced genomics tools has provided a great opportunity to analyze the entire gene set of all strains of a species, which is defined as the pangenome $[66,67]$. Interestingly, 


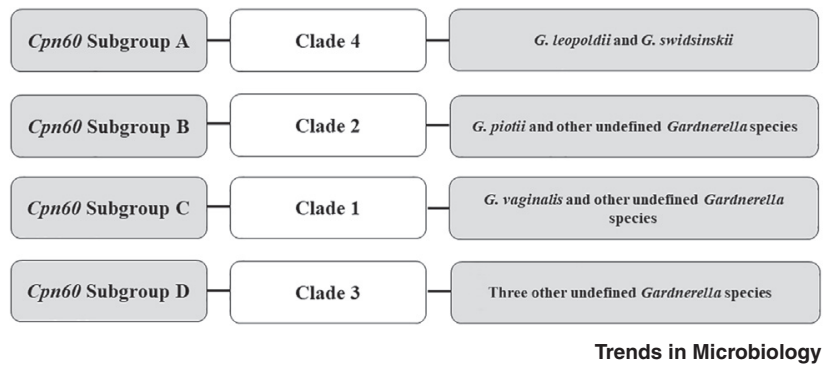

Figure 1. Association between Data Provided by Whole-Genome Sequencing. Classification of Gardnerella spp. based on cpn60 subgroups [59], on the clade system [48], and, lastly, the description of novel species [30].

congruent to the genomic characterization of Gardnerella spp. from the reproductive tract, isolates from bladders of women with symptoms of urgency urinary incontinence reveal a large pangenome [68]. Notably, Malki and colleagues showed that prophage gene sequences were found to be abundant amongst Gardnerella isolated from the bladder and from the vagina and endometrium [68]. Interestingly, examination of the coding sequences for all the investigated genomes failed to detect genes that were exclusive to the isolates from the bladder. These findings have highlighted indications that strongly suggest substantial horizontal gene transfer. In addition, bioinformatic evidence indicated that prophage acquisition is ongoing within both vaginal and bladder Gardnerella populations. Phages have been found to play a crucial role in the structuring of microbial communities, including those residing within the human body [69], driving bacterial genetic diversity [70] and adaptation to changes in the environment [71]. It has been postulated that lysogenic lactobacilli phages could enter the lytic cycle, contributing to a shift in the vaginal microbiota leading to BV $[72,73]$. Similarly, the abundance of prophage sequences within the analyzed Gardnerella strains suggests that phages could play an important role in the evolutionary history of the species, and in its interactions within the complex communities found in the female urinary and reproductive tracts. However, to date, no lytic phage specific for Gardnerella has been isolated.

\section{Gardnerella spp. Ecotypes}

It is critical to embrace the diversity of Gardnerella spp. to gain meaningful insight into its ecology [74]. An ecotype is a subspecies group in which the constituents are ecologically distinct from other members of the species [75]. Ecotypes thus represent lineages within species that possess unique adaptations and ecological capacities $[74,75]$. Noticeably, Cornejo and colleagues pointed out the existence of three major ecotypes of Gardnerella spp. based on the phylogenetic structure of their core and accessory genes and the cohesiveness in functional gene composition with ecotypes. Interestingly, only the genomes of isolates belonging to ecotype 1 encode several glycosidases (e.g., galactosidases, glucosidases, and fucosidases) and have expanded capabilities for galactoses and pentose sugar metabolism. Ecotype 2 is better characterized by the possession of at least two distinct genes encoding sialidase. Conversely, the majority of the genomes in ecotype 3 lack genes for any of these enzymes [74]. This is especially noteworthy considering the observations of Balashov and colleagues [17] that isolates identified as lineages showing an under-representation of sialidases were more prevalent among asymptomatic subjects. Taken together, these findings could be useful to more accurately identify and characterize lineages of Gardnerella spp. associated with symptoms of BV, ultimately improving diagnostic procedures for the disease.

\section{Functional Analysis of Virulence Potential of Gardnerella spp. Based on in vitro Assays}

Despite the recent findings provided by employing higher-resolution approaches, a considerable number of culture-based studies have shown the wide variety of functional features observed for Gardnerella spp. isolates in terms of ability to displace beneficial Lactobacillus spp., antimicrobial tolerance, biofilm formation, cytotoxicity, and adhesion to epithelial cells, as summarized in Table 3. 
Table 3. In vitro Studies of Functional Virulence Properties of Strains of Gardnerella Isolated from Women with a Clinical Diagnosis of BV versus Women in Whom BV Has Been Clinically Ruled Out

\begin{tabular}{|c|c|c|c|}
\hline Type of assay & $\begin{array}{l}\text { Number of } \\
\text { isolates }(n)\end{array}$ & Main conclusion & Refs \\
\hline \multicolumn{4}{|l|}{ Displacement of beneficial lactobacilli } \\
\hline $\begin{array}{l}\text { Gardnerella spp. ability to } \\
\text { induce displacement of lactobacilli } \\
\text { preadhered to epithelial cells }\end{array}$ & $n=14$ & $\begin{array}{l}\text { BV isolates had a stronger } \\
\text { ability to cause displacement of } \\
\text { Lactobacillus crispatus than } \\
\text { asymptomatic/non-BV }\end{array}$ & {$[81]$} \\
\hline \multicolumn{4}{|l|}{ Antimicrobial tolerance } \\
\hline $\begin{array}{l}\text { Minimal inhibitory concentration } \\
\text { (MIC) assays }\end{array}$ & $n=43$ & $\begin{array}{l}\text { Isolates from asymptomatic/ } \\
\text { non-BV women were also resistant } \\
\text { to metronidazole }\end{array}$ & {$[44]$} \\
\hline MIC assays & $n=6$ & $\begin{array}{l}\text { Isolates from both asymptomatic/ } \\
\text { non-BV and BV women were tolerant } \\
\text { to host immune components }\end{array}$ & {$[86]$} \\
\hline \multicolumn{4}{|l|}{ Biofilm formation } \\
\hline $\begin{array}{l}\text { Biofilm formation in 96-well } \\
\text { plates }\end{array}$ & $n=34$ & $\begin{array}{l}\text { The differences found in biofilm } \\
\text { formation between BV and } \\
\text { asymptomatic/non-BV isolates } \\
\text { were not statistically different }\end{array}$ & {$[51]$} \\
\hline $\begin{array}{l}\text { Biofilm formation in } 96 \text { well- } \\
\text { plates }\end{array}$ & $n=9$ & $\begin{array}{l}\text { Isolates from all four subgroups } \\
\text { produced biofilm }\end{array}$ & {$[42]$} \\
\hline \multicolumn{4}{|l|}{ Cytotoxicity } \\
\hline $\begin{array}{l}\text { Cytotoxicity to HeLa } \\
\text { epithelial cell }\end{array}$ & $n=6$ & $\begin{array}{l}\text { Gardnerella strains isolated } \\
\text { from women with BV were able } \\
\text { to induce more extensive } \\
\text { damage on the HeLa monolayer } \\
\text { than strains isolated from } \\
\text { asymptomatic/non-BV women }\end{array}$ & {$[85]$} \\
\hline \multicolumn{4}{|c|}{ Adherence, cytotoxicity, biofilm formation, and antimicrobial tolerance } \\
\hline $\begin{array}{l}\text { Adherence/cytotoxicity to ME-180 } \\
\text { epithelial cells }\end{array}$ & $n=2$ & $\begin{array}{l}\text { BV isolate was able to adhere } \\
\text { to larger numbers and cause } \\
\text { more cytotoxic effects } \\
\text { to ME-180 epithelial cells }\end{array}$ & {$[42]$} \\
\hline MIC assays and biofilm formation & & $\begin{array}{l}\text { Both isolates exhibited similar } \\
\text { antimicrobial tolerance to the } \\
12 \text { antibiotics tested; BV } \\
\text { isolates showed a high biofilm- } \\
\text { forming capacity }\end{array}$ & \\
\hline $\begin{array}{l}\text { Adherence/cytotoxicity to HeLa } \\
\text { epithelial cells }\end{array}$ & $n=14$ & $\begin{array}{l}\text { BV isolates were able to adhere } \\
\text { to larger numbers and cause } \\
\text { more cytotoxic effects to } \\
\text { HeLa cells; }\end{array}$ & [81] \\
\hline
\end{tabular}


Table 3. Continued

\begin{tabular}{|l|l|l|}
\hline Type of assay & $\begin{array}{l}\text { Number of } \\
\text { isolates }(n)\end{array}$ & Main conclusion \\
\hline MIC assays and biofilm formation & & $\begin{array}{l}\text { Roth isolates exhibited similar } \\
\text { antimicrobial tolerance to }\end{array}$ \\
\hline & $\begin{array}{l}\text { the } 12 \text { antibiotics tested; no } \\
\text { statistical differences were } \\
\text { found in biofilm formation }\end{array}$ \\
\hline
\end{tabular}

As stated at the beginning of this review, a hallmark of BV is the presence of a thick biofilm on vaginal epithelial cells [12,76-78]. The association of the biofilm phenotype to BV development raised an important question: do all Gardnerella spp. have the ability to develop a biofilm? In other biofilmassociated diseases it has been already established that not all strains within the same species form biofilms [79]. Evaluation of the biofilm-forming ability of two strains of Gardnerella spp., one isolated from a woman with a clinical diagnosis of BV and the other from a woman in whom BV had been clinically ruled out, showed that the biofilm-forming ability was significantly higher in the BV isolate, which would reasonably be consistent with greater ability to tolerate antimicrobial treatments [47]. The authors of the study found that the sequences of a putative biofilm-associated protein (BAP) family gene were quite disparate in both isolates and that could potentially explain the differences in biofilm formation [47]. BAP proteins are large, cell-wall-anchored adhesins that can mediate both adherence to host cells and intercellular adherence, thus contributing to biofilm formation [80]. The analysis of this phenotypic feature in a larger array of Gardnerella spp. isolated from both groups of women would be needed to support its role and to understand such variations. In this sense, our research group carried out a study which analyzed the biofilm-forming capacity of a higher number of isolates, using the same in vitro model described by Harwich and colleagues [47]. However, contrary to their findings, we did not observe significant differences in the in vitro biofilm-forming capacity between the isolates belonging to both groups [81]. The biofilm-forming ability of isolates belonging to the four Gardnerella subgroups, as proposed by Ahmed and colleagues [48], was also tested [42,51]. These studies also demonstrated no statistical differences in biofilm-forming capacity between the subgroups $[42,51]$. While microtiter plate assays have greatly increased our understanding of the biology of vaginal biofilms, it is becoming increasingly apparent that the in vitro methods do not accurately represent in vivo conditions and are highly sensitive to technical variability $[42,47,81]$. In vitro models have some drawbacks and shortcomings owing to the fact that the growth medium might not contain all the factors found in vivo, and some in vivo cues may turn on expression of biofilm-related genes. As such, by using such in vitro models, the process involved in the initial attachment to the substratum, which corresponds to the first stage of the biofilm formation, might be different from what happens in vivo [82-84].

Trying to overcome the limitations of the in vitro models and to better understand the differences between the isolates from asymptomatic/BV-negative or BV-positive women, our research group carried out some experiments using epithelial cells that could mimic the vaginal epithelium. Remarkably, using these conditions, Gardnerella spp. isolated from women with BV revealed a higher ability to cause cytotoxic effects on epithelial cells, as well as a higher ability to displace preadherent healthy vaginal lactobacilli, and, subsequently, achieving higher initial adhesion rates [81]. Biofilm formation under these conditions was unsuccessful due to strong cytotoxicity of Gardnerella that induced the death of the cell lines within $3 \mathrm{~h}$ of contact. Taken together, these findings support the most recent model of BV development [78], which suggested that the virulent Gardnerella spp. might be responsible for the trigger for BV development during the early stages of biofilm formation $[47,81,85]$.

Apart from the findings related to the different pathogenicity of Gardnerella spp. isolates $[44,47,81]$, another important insight providing evidence that not all strains have the same virulence potential was derived from a work conducted by Swidsinski and colleagues. They highlighted the importance 
of Gardnerella spp. biofilms when they observed that only biofilm-forming isolates were present in the sex partners of women with BV [76]. These findings led them to propose that the mere presence of loosely adherent Gardnerella spp. on the vaginal epithelium had a lesser clinical significance and that BV was sexually transmissible only in the presence of high-density clusters (biofilms) of Gardnerella spp. [76], which correlates with our findings [81]. In sum, all these studies support the hypothesis that certain members within the genus Gardnerella are less likely to induce BV, whereas others are more virulent and more likely to cause BV [78]. A weakness of such studies, however, is that the whole metagenomic community profiles from which samples tested in vitro are isolated, are often not analyzed. Whole metagenomic analysis would reveal the presence of strains that were not isolated. In the absence of whole metagenomic analysis, the possibility cannot be ruled out that clinical outcome is related to a strain of bacteria that was not isolated from the sample.

\section{Concluding Remarks}

This review summarizes current knowledge concerning the Gardnerella spp. physiology and molecular biology, and highlights the need to revise the bulk of original research of the past decades that considered all Gardnerella isolates as belonging to $G$. vaginalis. There remains a great deal of work to be done in elucidating the basic biology and metabolism of different Gardnerella species and their impact in BV development. It has been suggested that the functional role played by Gardnerella spp. within the vaginal microbiota could differ significantly depending on the subgroup(s) or species dominating the BV-type microbiota $[30,37]$. Therefore, there are gaps in knowledge regarding the role of Gardnerella spp. in the vaginal microbiome in eubiotic and dysbiotic states (see Outstanding Questions). Furthermore, it is also essential to carry out an array of phenotypic and genotypic studies to facilitate the correct classification of Gardnerella isolates and to find markers that are associated with BV. Research using a robust animal model could advance our understanding of BV pathogenesis, especially with regard to factors such as the role of biofilms, transmission mechanisms, and adverse pregnancy outcomes.

\section{Acknowledgments}

J.C. and N.C. acknowledge the projects PTDC/BIA-MIC/28271/2017 (POCl-01-0145-FEDER-028271), and the strategic funding of unit UID/BIO/04469/2019, supported by the Portuguese Foundation for Science and Technology.

\section{References}

1. Kenyon, C. et al. (2013) The global epidemiology of bacterial vaginosis: a systematic review. Am. J. Obstet. Gynecol. 209, 505-523

2. Bagnall, P. and Rizzolo, D. (2017) Bacterial vaginosis: a practical review. J. A. A. P. A. 30, 15-21

3. Peebles, K. et al. (2019) High global burden and costs of bacterial vaginosis: a systematic review and metaanalysis. Sex. Transm. Dis. 46, 304-311

4. Nasioudis, D. et al. (2017) Bacterial vaginosis: a critical analysis of current knowledge. B. J. O. G 124, 61-69

5. Findley, K. et al. (2016) Health disparities and the microbiome. Trends Microbiol. 24, 847-850

6. Onderdonk, A.B. et al. (2016) The human microbiome during bacterial vaginosis. Clin. Microbiol. Rev. 29, 223-238

7. Kaambo, E. et al. (2018) Vaginal microbiomes associated with aerobic vaginitis and bacterial vaginosis. Front. Public Health 6, 78

8. Greenbaum, S. et al. (2019) Ecological dynamics of the vaginal microbiome in relation to health and disease. Am. J. Obstet. Gynecol. 220, 324-335

9. Fredricks, D.N. et al. (2007) Targeted PCR for detection of vaginal bacteria associated with bacterial vaginosis. J. Clin. Microbiol. 45, 3270-3276

10. Eschenbach, D.A. et al. (1988) Diagnosis and clinical manifestations of bacterial vaginosis. Am. J. Obstet. Gynecol 158, 819-828
11. Amsel, R. et al. (1983) Nonspecific vaginitis: diagnostic criteria and microbial and epidemiologic associations. Am. J. Med 74, 14-22

12. Swidsinski, A. et al. (2005) Adherent biofilms in bacterial vaginosis. Obstet. Gynecol. 106, 1013-1023

13. Shimaoka, M. et al. (2019) Association between preterm delivery and bacterial vaginosis with or without treatment. Sci. Rep. 9, 509

14. Jacobsson, B. et al. (2002) Bacterial vaginosis in early pregnancy may predispose for preterm birth and postpartum endometritis. Acta Obstet. Gynecol. Scand. 81, 1006-1010

15. Judlin, P. (2010) Current concepts in managing pelvic inflammatory disease. Curr. Opin. Infect. Dis. 23, 83-87

16. Abdool Karim, S.S. et al. (2019) The genital tract and rectal microbiomes: their role in HIV susceptibility and prevention in women. J. Int. AIDS Soc. 22, e25300

17. Balashov, S.V. et al. (2014) Identification, quantification and subtyping of Gardnerella vaginalis in noncultured clinical vaginal samples by quantitative PCR. J. Med. Microbiol. 63, 162-175

18. Srinivasan, S. et al. (2010) Temporal variability of human vaginal bacteria and relationship with bacterial vaginosis. PLoS One 5, e10197

19. Leopold, S. (1953) Heretofore undescribed organism isolated from the genitourinary system. U. S. Armed Forces Med. J. 4, 263

\section{Outstanding Questions}

Do certain lineages or species of Gardnerella vary in their virulence potential according to the BV status? Can Gardnerella spp. be opportunistic pathogens, present in the vaginal microbiota that, under specific conditions, might turn into a more virulent state? Are bacteriophages involved?

Do ecological interactions between different Gardnerella species that colonize the same woman enhance virulence potential and, consequently, clinical outcome?

Relative to Gardnerella isolates from women in whom BV has been clinically ruled out, do isolates from women with a clinical diagnosis of BV differ in their susceptibility to antimicrobial agents, and is this related to the recurrence of BV? 
20. Gardner, H.L. and Dukes, C.D. (1955) Haemophilus vaginalis vaginitis: a newly defined specific infection previously classified non-specific vaginitis. Am. J. Obstet. Gynecol. 69, 962-976

21. Zinnemann, K. and Turner, G. (1963) The taxonomic position of 'Haemophilus vaginalis' [Corynebacterium vaginale]. J. Pathol. Bacteriol. 85, 213-219

22. Greenwood, J.R. and Pickett, M.J. (1980) Transfer of Haemophilus vaginalis Gardner and Dukes to a new genus, Gardnerella: G. vaginalis (Gardner and Dukes) comb. nov. Int. J. Syst. Evol. Microbiol. 30, 170-178

23. Piot, P. et al. (1980) A taxonomic study of Gardnerella vaginalis (Haemophilus vaginalis) Gardner and Dukes 1955. J. Gen. Microbiol. 119, 373-396

24. Catlin, B.W. (1992) Gardnerella vaginalis: characteristics, clinical considerations, and controversies. Clin. Microbiol. Rev. 5, 213-237

25. Sadhu, K. et al. (1989) Gardnerella vaginalis has a Gram-positive cell-wall ultrastructure and lacks classical cell-wall lipopolysaccharide. J. Med. Microbiol. 29, 229-235

26. Scott, T.G. et al. (1989) Electron microscopy of adhesive interactions between Gardnerella vaginalis and vaginal epithelial cells, McCoy cells and human red blood cells. J. Gen. Microbiol. 135, 475-480

27. Cereija, T.B. et al. (2013) Influence of anaerobic conditions on vaginal microbiota recovery from bacterial vaginosis patients. Sex. Transm. Infect. 89 307

28. Malone, B.H. et al. (1975) Obligately anaerobic strains of Corynebacterium vaginale (Haemophilus vaginalis). J. Clin. Microbiol. 2, 272-275

29. Calasanti, T.M. (1996) Incorporating diversity: meaning, levels of research, and implications for theory. Gerontologist 36, 147-156

30. Vaneechoutte, M. et al. (2019)

Emended description of Gardnerella vaginalis and description of Gardnerella leopoldii sp. nov. Gardnerella piotii sp. nov. and Gardnerella swidsinskii sp. nov., with delineation of 13 genomic species within the genus Gardnerella. Int. J. Syst. Evol. Microbiol. 69, 679-687

31. Janda, J.M. and Abbott, S.L. (2007) 16S rRNA gene sequencing for bacterial identification in the diagnostic laboratory: pluses, perils, and pitfalls. J. Clin. Microbiol. 45, 2761-2764

32. Konstantinidis, K.T. et al. (2006) The bacterial species definition in the genomic era. Philos. Trans. R. Soc. Lond. B. Biol. Sci. 361, 1929-1940

33. Schwebke, J.R. et al. (2014) Role of Gardnerella vaginalis in the pathogenesis of bacterial vaginosis: a conceptual model. J. Infect. Dis. 210, 338-343

34. Hickey, R.J. and Forney, L.J. (2014) Gardnerella vaginalis does not always cause bacterial vaginosis. J. Infect. Dis. 210, 1682-1683

35. Ravel, J. et al. (2011) Vaginal microbiome of reproductive-age women. Proc. Natl. Acad. Sci. U.S. A. $108,4680-4687$

36. Schwebke, J.R. et al. (2014) Prevalence of Gardnerella vaginalis among women with Lactobacilluspredominant vaginal flora. Sex. Transm. Infect 90, 61-63

37. Schellenberg, J.J. et al. (2017) Gardnerella vaginalis diversity and ecology in relation to vaginal symptoms. Res. Microbiol. 168, 837-844

38. Piot, P. et al. (1984) Biotypes of Gardnerella vaginalis. J Clin Microbiol 20,677-679

39. Benito, R. et al. (1986) A modified scheme for biotyping Gardnerella vaginalis. J. Med. Microbiol. 21, 357-359

40. Pedraza-Aviles, A.G. et al. (1995) Gardnerella vaginalis biotypes: modification of a proposed system. Rev. Latinoam. Microbiol. 37, 19-26
41. Briselden, A.M. and Hillier, S.L. (1990) Longitudinal study of the biotypes of Gardnerella vaginalis. J. Clin. Microbiol. 28, 2761-2764

42. Jayaprakash, T.P. et al. (2012) Resolution and characterization of distinct cpn60-based subgroups of Gardnerella vaginalis in the vaginal microbiota. PLoS One 7, e43009

43. Pleckaityte, M. et al. (2012) Genetic and biochemical diversity of Gardnerella vaginalis strains isolated from women with bacterial vaginosis. FEMS Immunol. Med. Microbiol. 65, 69-77

44. Aroutcheva, A. et al. (2001) Gardnerella vaginalis isolated from patients with bacterial vaginosis and from patients with healthy vaginal ecosystems. Clin. Infect. Dis 33, 1022-1027

45. Huang, B. et al. (2014) The changing landscape of the vaginal microbiome. Clin. Lab. Med. 34, 747-761

46. Yeoman, C.J. et al. (2010) Comparative genomics of Gardnerella vaginalis strains reveals substantial differences in metabolic and virulence potential. PLoS One 5, e12411

47. Harwich, M. et al. (2010) Drawing the line between commensal and pathogenic Gardnerella vaginalis through genome analysis and virulence studies. BMC Genomics 11, 375

48. Ahmed, A. et al. (2012) Comparative genomic analyses of 17 clinical isolates of Gardnerella vaginalis provide evidence of multiple genetically isolated clades consistent with subspeciation into genovars. J. Bacteriol. 194, 3922-3937

49. Lopes dos Santos Santiago, G et al. (2011) Gardnerella vaginalis comprises three distinct genotypes of which only two produce sialidase. Am. J. Obstet. Gynecol. 204, 450. e1-450. e7

50. Hardy, L. et al. (2017) The presence of the putative Gardnerella vaginalis sialidase A gene in vaginal specimens is associated with bacterial vaginosis biofilm. PLoS One 12, e0172522

51. Janulaitiene, M. et al. (2018) Phenotypic characterization of Gardnerella vaginalis subgroups suggests differences in their virulence potential. PLoS One 13, e0200625

52. Gelber, S.E. et al. (2008) Functional and phylogenetic characterization of vaginolysin, the human-specific cytolysin from Gardnerella vaginalis. J. Bacteriol. 190, 3896-3903

53. Zilnyte, M. et al. (2015) The cytolytic activity of vaginolysin strictly depends on cholesterol and is potentiated by human CD59. Toxins 7, 110-128

54. Lewis, W.G. et al. (2013) Degradation, foraging, and depletion of mucus sialoglycans by the vaginaadapted Actinobacterium Gardnerella vaginalis. J. Biol. Chem. 288, 12067-12079

55. Lewis, A.L. and Lewis, W.G. (2012) Host sialoglycans and bacterial sialidases: a mucosal perspective. Cell Microbiol. 14, 1174-1182

56. Robinson, L.S. et al. (2019) Identification and characterization of $\mathrm{NanH} 2$ and $\mathrm{NanH} 3$, enzymes responsible for sialidase activity in the vaginal bacterium Gardnerella vaginalis. J. Biol. Chem. 294, 5230-5245

57. Hill, J.E. et al. (2005) Characterization of vaginal microflora of healthy, nonpregnant women by chaperonin-60 sequence-based methods. Am. J. Obstet. Gynecol. 193, 682-692

58. Schellenberg, J.J. et al. (2011) Molecular definition of vaginal microbiota in East African commercial sex workers. Appl. Environ. Microbiol. 77, 4066-4074

59. Schellenberg, J.J. et al. (2016) Gardnerella vaginalis subgroups defined by cpn60 sequencing and sialidase activity in isolates from Canada, Belgium and Kenya. PLoS One 11, e0146510

60. Janulaitiene, M. et al. (2017) Prevalence and distribution of Gardnerella vaginalis subgroups in 
women with and without bacterial vaginosis. BMC Infect. Dis. 17, 394

61. Hilbert, D.W. et al. (2017) Gardnerella vaginalis population dynamics in bacterial vaginosis. Eur. J. Clin. Microbiol. Infect. Dis. 36, 1269-1278

62. Shipitsyna, E. et al. (2019) Quantitation of all four Gardnerella vaginalis clades detects abnormal vaginal microbiota characteristic of bacterial vaginosis more accurately than putative $G$. vaginalis sialidase A gene count. Mol. Diagn. Ther. 23, 139-147

63. Hill, J.E. et al. (2019) Resolution and co-occurrence patterns of Gardnerella leopoldii, Gardnerella swidsinskii, Gardnerella piotii and Gardnerella vaginalis within the vaginal microbiome. Infect. Immun. Published online September 16, 2019. https://doi.org/10.1128/IAl.00532-19

64. Albert, A.Y. et al. (2015) A study of the vaginal microbiome in healthy Canadian women utilizing cpn60-based molecular profiling reveals distinct Gardnerella subgroup community state types. PLoS One 10, e0135620

65. Vodstrcil, L.A. et al. (2017) The influence of sexua activity on the vaginal microbiota and Gardnerella vaginalis clade diversity in young women. PLoS One 12, e017185666

66. Medini, D. et al. (2005) The microbial pan-genome. Curr. Opin. Genet. Dev. 15, 589-594

67. Vernikos, G. et al. (2015) Ten years of pangenome analyses. Curr. Opin. Microbiol. 23, 148-154

68. Malki, K. et al. (2016) Genomes of Gardnerella strains reveal an abundance of prophages within the bladder microbiome. PLoS One 11, e0166757

69. Clokie, M.R. et al. (2011) Phages in nature. Bacteriophage 1, 31-45

70. Koskella, B. and Brockhurst, M.A. (2014) Bacteriaphage coevolution as a driver of ecological and evolutionary processes in microbial communities. FEMS Microbiol. Rev. 38, 916-931

71. Roberts, A.P. and Kreth, J. (2014) The impact of horizontal gene transfer on the adaptive ability of the human oral microbiome. Front. Cell Infect. Microbiol. 4,124

72. Martin, R. et al. (2010) Induction, structural characterization, and genome sequence of Lv1, a prophage from a human vaginal Lactobacillus jensenii strain. Int. Microbiol. 13, 113-121
73. Miller-Ensminger, T. et al. (2018) Bacteriophages of the urinary microbiome. J. Bacteriol. 200, e00738-17.

74. Cornejo, O.E. et al. (2018) Focusing the diversity of Gardnerella vaginalis through the lens of ecotypes. Evol. Appl. 11, 312-324

75. Cohan, F.M. (2006) Towards a conceptual and operational union of bacterial systematics, ecology, and evolution. Philos. Trans. R. Soc. Lond. B. Biol. Sci. 361, 1985-1996

76. Swidsinski, A. et al. (2010) Gardnerella biofilm involves females and males and is transmitted sexually. Gynecol. Obstet. Invest. 70, 256-263

77. Machado, D. et al. (2015) Bacterial vaginosis biofilms: challenges to current therapies and emerging solutions. Front. Microbiol. 6, 1528

78. Muzny, C.A. et al. (2019) An updated conceptual model on the pathogenesis of bacterial vaginosis. J. Infect. Dis. 220, 1399-1405

79. Lianou, A. and Koutsoumanis, K.P. (2013) Strain variability of the behavior of foodborne bacterial pathogens: a review. Int. J. Food. Microbiol. 167, 310-321

80. Lasa, I. and Penades, J.R. (2006) Bap: a family of surface proteins involved in biofilm formation. Res. Microbiol. 157, 99-107

81. Castro, J. et al. (2015) Using an in-vitro biofilm model to assess the virulence potential of bacterial vaginosis or non-bacterial vaginosis Gardnerella vaginalis isolates. Sci. Rep. 5, 11640

82. Roberts, A.E. et al. (2015) The limitations of in vitro experimentation in understanding biofilms and chronic infection. J. Mol. Biol. 427, 3646-3661

83. Magana, M. et al. (2018) Options and limitations in clinical investigation of bacterial biofilms. Clin. Microbiol. Rev. 31, e00084-16.

84. Azeredo, J. et al. (2017) Critical review on biofilm methods. Crit. Rev. Microbiol. 43, 313-351

85. Castro, J. et al. (2018) Lactobacillus crispatus represses vaginolysin expression by BV associated Gardnerella vaginalis and reduces cell cytotoxicity. Anaerobe 50, 60-63

86. Castro, J. et al. (2018) Innate immune components affect growth and virulence traits of bacterialvaginosis-associated and non-bacterial-vaginosis associated Gardnerella vaginalis strains similarly. Pathog. Dis. 76, fty089 